\title{
LESSON 15
}

\section{MARGINS:}

Left, Pica 20; Elite 30;

Right, moved out of the way.

The $f$ sign

(E)

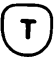

(F)

Check the 'ready-to-type' position.

The hyphen (-) key
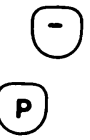

Line-end division of words

Read these rules and those for the margin release before typing this paragraph.

\section{MARGINS:}

Pica 20-70; Elite 30-70.

Keep the right margin as level as possible.

\section{The Bell and the Margin Release}

Practise line-end division

Type this paragraph with the margins indicated above.
Practise the reach until you can type $f$ without looking down, using the $f$ finger and the right shift key. Keep the a finger in position.

Leave one space before a $£$ sign but no space after it, after this first line.

Type these lines as indicated by your teacher. Keep your eyes on the copy.

1. fff ft5 ft£ $5 £ 5$ f£5 f£5 f£5f ft£ fff£ ftf ff5f fff

2. Send me $£ l$, Fred $£ 4$, Tony $£ 5$, Eric $£ 5$ and Mark $£ l$.

3. Count up the $£ l, £ 5$ and $£ l 0$ notes into $f l o O$ piles.

If the hyphen is not above the $p$ key on the top row, your teacher will give you the first line and you should ignore line 4. Practise the reach until you can type the hyphen without looking down. Do not leave a space before or after the hyphen.

Type these lines as indicated by your teacher.

4. $;$; ; ip; ip- ip- ;-- --- ;-; i-p; ;--i p-p p-- i--i

5. to-do; co-opt; cloak-room; post-date; co-education

6. Vale-Price will be his father-in-law later to-day.

When you type, the machine keeps the margin on the left straight if you return the carriage properly but you must keep the margin on the right straight as far as possible. The breaking up of words at the end of a line by means of the hyphen will help you do this.

1. Never divide a word unless you must. If you must divide:

2. Never divide a word which starts with 1. Place the hyphen directly after the a capital letter unless the word is first part of the word you are dividing, hyphenated, in which case divide e.g., rush-ing.

where the hyphen occurs.

3. Never divide numbers, sums of money or dates, e.g., $£ 1,560$ or 1918 .

4. Never divide a word of one syllable, e.g., though.

5. Never divide abbreviations - e.g.

6. Never divide after only 2 letters, e.g., 4. As a general rule, do not divide the divi-sion not di-vision.

2. Divide with at least 3 letters of the word remaining.

3. Divide between syllables so that the pronunciation of the word being divided is understood from the first line, e.g., pro-gramme.

first and last lines of a paragraph or divide on consecutive lines.

If you set a margin on the right you will find that a bell will ring to warn you that you are nearing the end of the line. You will have 5 or 6 spaces in which to decide if you can finish the word on that line or divide it. One of the uses of the margin release is that it enables you to unlock the right-hand margin and so finish a word you must not divide. Your teacher will tell you where the margin release key is. When you unlock the margin by pressing the margin release key, you unlock the margin for that particular line only.

\section{LESSON 15}

